\title{
E2 Strength in the Radiative Charmed Baryon Decay $\Sigma_{c}^{*} \rightarrow \Lambda_{c} \gamma^{\dagger}$
}

\author{
Martin J. Savage \\ Department of Physics, Carnegie Mellon University, Pittsburgh PA 15213
}

\begin{abstract}
The radiative decay $\Sigma_{Q}^{*} \rightarrow \Lambda_{Q} \gamma$ can have both magnetic dipole (M1) and electric quadrupole (E2) components. In the heavy quark limit $M_{Q} \rightarrow \infty$ the transition arises from the spin of the light degrees of freedom changing from $s_{l}=1$ to $s_{l}=0$ and hence the E2 contribution vanishes. We compute the leading contribution to the E2 strength in chiral perturbation theory and find that the amplitude is enhanced by a small energy denominator in the chiral limit. This enhancement essentially compensates for the $1 / M_{c}$ suppression that is present in the charm system. We find a mixing ratio of order a few percent dependent upon the $\Sigma_{c^{-}} \Sigma_{c}^{*}$ spin symmetry breaking mass difference. The analogous quantity in the b-baryon sector is smaller by a factor of $\sim M_{c} / M_{b}$.
\end{abstract}

CMU-HEP 94-25

DOE-ER/40682-79

hep-ph/9408294

August 1994

\footnotetext{
$\dagger$ Work supported in part by the Department of Energy under contract DE-FG0291ER40682.
} 
It has recently been shown that the leading contribution to the electric quadrupole (E2) strength of a radiative transition between light baryons, such as $\Delta \rightarrow N \gamma$, can be computed in chiral perturbation theory [1]. It was found that such matrix elements are enhanced in the chiral limit by factors of $\sim \log \left(m_{\pi}^{2} / \Lambda_{\chi}^{2}\right)$ (where $\Lambda_{\chi}$ is the chiral symmetry breaking scale) giving mixing ratios (ratio of E2 to M1 matrix elements) typically of order a few percent.

The situation is somewhat more complicated for transitions between baryons containing a single heavy quark. In the limit that the heavy quark mass is infinitely greater than the scale of strong interactions $\left(M_{Q} \gg \Lambda_{\mathrm{QCD}}\right)$ the lowest lying baryons containing one heavy quark can be classified by the spin of the light degrees of freedom. In the charm systems, the $\overline{\mathbf{3}}$ of charmed baryons $\left(\Lambda_{c}^{+}, \Xi_{c 3}^{+}\right.$and $\left.\Xi_{c 3}^{0}\right)$ have the light degrees of freedom in a spin zero configuration $\left(s_{l}=0\right)$ while the $6^{(*)}$ of charmed baryons $\left(\Sigma_{c}^{++(*)}, \Sigma_{c}^{+(*)}, \Sigma_{c}^{0(*)}\right.$ , $\Xi_{c 6}^{+(*)}, \Xi_{c 6}^{0(*)}$ and $\left.\Omega_{c}^{0(*)}\right)$ have the light degrees of freedom in a spin one configuration $\left(s_{l}=1\right)$.

The radiative decay $\Sigma_{Q}^{*} \rightarrow \Lambda_{Q} \gamma(Q=c, b)$ involves a $J=\frac{3}{2}$ state with $s_{l}=1$ decaying into a $J=\frac{1}{2}$ state with $s_{l}=0$. The heavy quark is a spectator during the transition and the decay proceeds entirely through the change of spin of the light degrees of freedom, allowing only magnetic dipole (M1) radiation. It is clear that any E2 radiation can only arise from finite mass effects of the heavy quark. Naively this suggests that the E2/M1 mixing ratio $\delta$ will be much smaller in heavy baryons than the corresponding quantity in the light baryon sector. However, we will show that in fact the E2 matrix element is enhanced by a small energy denominator in the chiral limit that essentially compensates for the $1 / M_{c}$ suppression in the charmed baryon sector. A thorough investigation of the M1 radiative decays of charmed baryons can be found in [2] [3] but there has been no discussion of the contribution from higher multipoles where appropriate. With the fixed target experiment E781 scheduled to run in 1996 that is capable of measuring the angular distribution of the photons in charmed baryon decays it seems timely to try and better understand such processes. In this work we will compute the leading contribution to the E2 matrix elements for $\Sigma_{Q}^{*} \rightarrow \Lambda_{Q} \gamma$ in chiral perturbation theory.

The strong interaction dynamics of quarks greatly simplifies in the limit that their mass becomes infinitely greater than the scale of strong interactions [4]- [6]. The new symmetries that become manifest in this limit have been been combined with chiral symmetry order to describe the soft hadronic interactions of hadrons containing a single heavy quark [7]-10] (for a review see [11] ). The lowest lying $\overline{\mathbf{3}}$ of baryons containing a heavy quark is 
described by the field $T_{i}(v)$ where $v$ is the four-velocity of the baryon (conserved during soft hadronic interactions) and where $i$ is the $\mathrm{SU}(3)$ index. Similarly, the lowest lying $6^{(*)}$ of baryons containing a heavy quark is denoted by the field $S(v)_{\mu}^{i j}$ (symmetric on $i, j$ ) where $i, j$ are $\mathrm{SU}(3)$ indices and where $\mu$ is a lorentz index. The chiral lagrangian describing the lowest order soft hadronic interactions of these baryons is given by (using the notation of [10] )

$$
\begin{aligned}
\mathcal{L}= & \bar{T}^{i} i v \cdot D T_{i}-\bar{S}_{i j}^{\mu} i v \cdot D S_{\mu}^{i j}+\Delta_{0} \bar{S}_{i j}^{\mu} S_{\mu}^{i j} \\
& +g_{3}\left(\epsilon_{i j k} \bar{T}^{i}\left(A^{\mu}\right)_{l}^{j} S_{\mu}^{k l}+\text { h.c. }\right)+i g_{2} \epsilon_{\mu \nu \rho \sigma} \bar{S}_{i j}^{\mu} v^{\nu}\left(A^{\rho}\right)_{k}^{i} S^{\sigma, j k}
\end{aligned}
$$

The axial field of pseudo-Goldstone bosons $A_{\mu}=\frac{i}{2}\left(\xi \partial_{\mu} \xi^{\dagger}-\xi^{\dagger} \partial_{\mu} \xi\right)$ is defined in terms of $\xi=\exp (i M / f)$ where $M$ is the octet of pseudo-Goldstone bosons represented by

$$
M=\left(\begin{array}{ccc}
\eta / \sqrt{6}+\pi^{0} / \sqrt{2} & \pi^{+} & K^{+} \\
\pi^{-} & \eta / \sqrt{6}-\pi^{0} / \sqrt{2} & K^{0} \\
K^{-} & \bar{K}^{0} & -2 \eta / \sqrt{6}
\end{array}\right)
$$

The residual mass $\Delta_{0}$ (mass difference between the $\overline{\mathbf{3}}$ and $\mathbf{6}$ ) is present even in the infinite mass limit and arises from the energy difference between the light degrees of freedom in the $\overline{\mathbf{3}}$ and $\mathbf{6}$. Using the notation of [10 we write

$$
\begin{aligned}
T_{i}(v) & =\frac{1}{2}(1+\psi) B_{i} \\
S_{\mu}^{i j}(v) & =\frac{1}{\sqrt{3}}\left(\gamma_{\mu}+v_{\mu}\right) \gamma_{5} \frac{1}{2}(1+\psi) B^{i j}+\frac{1}{2}(1+\psi) B_{\mu}^{* i j} .
\end{aligned}
$$

The charmed baryons in the $\overline{\mathbf{3}}$ have $\mathrm{SU}(3)$ assignments

$$
B_{1}=\Xi_{c 3}^{0}, \quad B_{2}=-\Xi_{c 3}^{+}, \quad B_{3}=\Lambda_{c}^{+},
$$

while the $J=1 / 2$ charmed baryons in the 6 have $\mathrm{SU}(3)$ assignments

$$
\begin{gathered}
B^{11}=\Sigma_{c}^{++}, \quad B^{12}=\frac{1}{\sqrt{2}} \Sigma_{c}^{+}, \quad B^{22}=\Sigma_{c}^{0} \\
B^{13}=\frac{1}{\sqrt{2}} \Xi_{c 6}^{+}, \quad B^{23}=\frac{1}{\sqrt{2}} \Xi_{c 6}^{0}, \quad B^{33}=\Omega_{c}^{0}
\end{gathered} .
$$

The $J=3 / 2$ members of the $\mathbf{6}^{*}$ have the same $\mathrm{SU}(3)$ assignments in $B_{\mu}^{* i j}$ as their $J=1 / 2$ partners have in $B^{i j}$. We will need the strong decay widths of the $\Sigma_{c}^{*+}$ later in this work and they can be shown to be

$$
\Gamma\left(\Sigma_{c}^{+} \rightarrow \Lambda_{c} \pi^{0}\right)=\Gamma\left(\Sigma_{c}^{*+} \rightarrow \Lambda_{c} \pi^{0}\right)=\frac{g_{3}^{2}}{6 \pi f_{\pi}^{2}}\left|\mathbf{k}_{\pi}\right|^{3}
$$


and

$$
\Gamma\left(\Sigma_{c}^{*+} \rightarrow \Sigma_{c} \pi\right)=\frac{g_{2}^{2}}{18 \pi f_{\pi}^{2}}\left|\mathbf{k}_{\pi}\right|^{3},
$$

where $\mathbf{k}_{\pi}$ is the pion three-momentum.

A complete discussion of the M1 decays of baryons containing a single heavy quark can be found in [2] [3]. The width for the decay $\Sigma_{Q}^{*} \rightarrow \Lambda_{Q} \gamma$ is given by

$$
\Gamma\left(\Sigma_{Q}^{*} \rightarrow \Lambda_{Q} \gamma\right)=\frac{E_{\gamma}^{3}}{12 \pi}\left[\left|A_{1}\right|^{2}+3\left|A_{2}\right|^{2}\right]
$$

where $A_{1}$ is the M1 amplitude, $A_{2}$ is the E2 amplitude and $E_{\gamma}$ is the energy of the emitted photon. The E2/M1 mixing ratio is defined by

$$
\delta=\frac{A_{2}}{A_{1}}
$$

In chiral perturbation theory the M1 matrix element is dominated by a dimension five local counter term,

$$
\mathcal{O}_{\mathrm{M} 1} \sim \frac{e}{\Lambda_{\chi}} \bar{\Lambda}_{Q} \mathcal{Q} \gamma^{\mu} \gamma_{5} \Sigma_{Q}^{* \nu} F_{\mu \nu},
$$

where $\mathcal{Q}$ is the electromagnetic charge matrix for the three light quarks given by

$$
\mathcal{Q}=\frac{1}{3}\left(\begin{array}{ccc}
2 & 0 & 0 \\
0 & -1 & 0 \\
0 & 0 & -1
\end{array}\right) \text {. }
$$

Loops and higher dimension operators are suppressed by additional powers of $\Lambda_{\chi}$. As this operator has an unknown coefficient and there is no experimental data on this decay mode we cannot yet determine the M1 matrix element. However, the M1 matrix element $A_{1}$ has been computed in the non-relativistic quark model (NRQM) [2] and is found to be

$$
A_{1}^{\mathrm{NRQM}}=-\frac{e}{M_{U}}
$$

where $M_{U}$ is the mass of a constituent up quark $\sim 300 \mathrm{MeV}$ (there is no contribution from a heavy quark interaction since the spin of the light degrees of freedom must change during the transition). The NRQM estimate should be relatively reliable as it generally reproduces well the value of M1 matrix elements corresponding to simply spin-flip (short distance) transitions. Of course, this is only a guide and ultimately the M1 matrix elements will be determined directly from experiment. More importantly, the NRQM is found to predict very small E2 amplitudes, as they arise from ground state configuration mixing 
(see for example [12]). In contrast, the estimates found in chiral perturbation theory are relatively large [1] and result from long-distance charged pion configurations (loops) that are not present in the NRQM.

The leading contribution to the E2 matrix element for $\Sigma_{Q}^{*} \rightarrow \Lambda_{Q} \gamma$ arises from the graph shown in fig. 1 . There is a cancellation between the $\Sigma_{Q}^{*}$ and $\Sigma_{Q}$ intermediate states that becomes exact as the states become degenerate. This is a result of the heavy quark spin symmetry which is broken by the $\Sigma_{Q}^{*}-\Sigma_{Q}$ mass splitting, denoted by $\Delta_{Q}$. At this order there is no contribution from $\Lambda_{Q}$ intermediate states as its coupling to pions is explicitly suppressed by $1 / M_{Q}$. We find that the E2 amplitude is given by

$$
A_{2}=\frac{2 \sqrt{2}}{3} \frac{e g_{2} g_{3}}{16 \pi^{2} f_{\pi}^{2}} F\left(\Delta_{Q}, E_{\gamma}, m_{\pi}\right)
$$

where the function $F(\Delta, E, m)$ is

$$
\begin{aligned}
F(\Delta, E, m) & =\int_{0}^{1} d x(1-2 x)[J(x E-\Delta, m)-J(x E, m)] \\
J(y, m) & =\sqrt{y^{2}-m^{2}} \log \left(\frac{y+\sqrt{y^{2}-m^{2}+i \epsilon}}{y-\sqrt{y^{2}-m^{2}+i \epsilon}}\right)
\end{aligned}
$$

In the limit that $\Delta \ll E_{\gamma}, m_{\pi}$ we can expand the function and find that

$$
F(\Delta, E, m)=\int_{0}^{1} d x x(1-2 x) \frac{E \Delta}{\sqrt{x^{2} E^{2}-m^{2}}} \log \left(\frac{x E+\sqrt{x^{2} E^{2}-m^{2}+i \epsilon}}{x E-\sqrt{x^{2} E^{2}-m^{2}+i \epsilon}}\right)
$$

which has the limits

$$
\begin{aligned}
F(\Delta, E, m) & \rightarrow \frac{\pi}{6} \frac{\Delta}{m_{\pi}} E \quad \text { for } E \ll m_{\pi} \\
& \rightarrow \frac{\Delta}{E} E \quad \text { for } E \gg m_{\pi}
\end{aligned}
$$

We see that the infrared divergence of the loop graph is regulated by the larger of $m_{\pi}$ and $E_{\gamma}$, giving a small energy denominator. We have kept a factor of $E$ in (16) because the E2 operator

$$
\mathcal{O}_{\mathrm{E} 2} \sim \frac{e}{\Lambda_{\chi}^{2}} \bar{\Lambda}_{Q} \mathcal{Q} \gamma^{\mu} \gamma_{5} \Sigma_{Q}^{* \nu} v^{\alpha}\left(\partial_{\mu} F_{\alpha \nu}+\mu \leftrightarrow \nu\right)
$$

is dimension six and when expanded has an explicit factor of $E_{\gamma} / \Lambda_{\chi}^{2}$. Naively one would expect this E2 operator to have a coefficient suppressed by a factor of $\Lambda_{\mathrm{QCD}} / M_{Q}$, giving a mixing ratio smaller than that found from the long-distance loop graph. 
We now turn to the charmed baryon sector and make an estimate of $\delta$ for $\Sigma_{c}^{*} \rightarrow \Lambda_{c} \gamma$ - The $\Sigma_{c}^{*}$ has not been observed to date and so the photon energy for the transition is an unknown, as is the size of the $\Sigma_{c}^{*}-\Sigma_{c}$ spin-symmetry breaking mass difference. Further, the axial coupling constants $g_{2,3}$ have not been determined experimentally. Recently, it has been shown that they are related to the $N N \pi$ coupling constant, $g_{A}=1.25$ in the large- $N_{C}$ limit of QCD ( $N_{C}$ is the number of colours) and we will use this estimate of $g_{3}=\sqrt{3 / 2} g_{A}$ and $g_{2}=-3 / 2 g_{A}$ [13] [14] in our computations. Using the NRQM estimate of the M1 strength and the large- $N_{c}$ values of $g_{2,3}$ we find that the radiative branching fraction is $\sim 1 \%$ for $M_{\Sigma_{c}^{*}}-M_{\Sigma_{c}}=40 \mathrm{MeV}$ and doesn't vary much over a reasonable range for $M_{\Sigma_{c}^{*}}-M_{\Sigma_{c}}$. The E2/M1 mixing ratio $\delta$ is shown in fig. 2 as a function of $M_{\Sigma_{c}^{*}}-M_{\Sigma_{c}}$ for the large- $N_{C}$ values of $g_{2}$ and $g_{3}$. When $M_{\Sigma_{c}^{*}}-M_{\Sigma_{c}}>m_{\pi}$ an imaginary part develops due to the presence of $\Sigma_{c} \pi$ physical intermediate states in the loop graph. We see from fig. 2 that an E2/M1 mixing ratio of order a percent or so is expected.

In order to determine $\delta$ in the radiative decay $\Sigma_{Q}^{*} \rightarrow \Lambda_{Q} \gamma$ the initial $\Sigma_{Q}^{*}$ 's must be aligned, by which we mean that the probablility of being in the $m= \pm \frac{3}{2}$ angular momentum states $w\left( \pm \frac{3}{2}\right)$ are different from the probablility of being in the $m= \pm \frac{1}{2}$ angular momentum states $w\left( \pm \frac{1}{2}\right)$. The resulting angular distribution of photons from the decay $\Sigma_{Q}^{*} \rightarrow \Lambda_{Q} \gamma$ of an aligned $\Sigma_{Q}^{*}$ has the form [1] 15]

$$
S(\theta)=1+\left[w\left( \pm \frac{3}{2}\right)-w\left( \pm \frac{1}{2}\right)\right] \frac{1+6 R e(\delta)-3|\delta|^{2}}{2\left(1+3|\delta|^{2}\right)} P_{2}(\cos \theta)
$$

where $P_{2}(x)$ is the second Legendre polynomial and $\theta$ is the angle between the alignment axis and the photon direction. At present the alignment of charmed baryons that will be produced in E781 cannot be predicted and will have to be determined experimentally. However, if the polarisations observed in the production of strange baryons are an indication of what we may expect in the charm sector then the $\Sigma_{c}^{*}$ s could have substantial alignment.

It must be remembered that we have computed only the leading contribution to the mixing ratio. There will be contributions from other $1 / M_{c}$ operators that are formally suppressed in the chiral and heavy quark limits but in reality may have a nonnegligible contribution. We therefore must regard this calculation as an estimate only and an example of an additional $1 / M_{c}$ contribution is from loop graphs with the $\Lambda_{c}$ as an intermediate state. Its coupling to pions is explicitly $1 / M_{c}$ and we expect the loop graph to be of order $\sim \log \left(m_{\pi}^{2} / \Lambda_{\chi}^{2}\right)$ for $E_{\gamma} \ll m_{\pi}$, this is less singular in the chiral limit than the loop graph 
we have computed (this graph does have an imaginery part for all values of $M_{\Sigma_{c}^{*}}-M_{\Sigma_{c}}$ as $\left.M_{\Sigma_{c}^{*}}-M_{\Lambda_{c}}>m_{\pi}\right)$. However, we know that $E_{\gamma} \sim m_{\pi}$ and these additional contributions may be significant. Our results, of course, can be applied to the b-baryons by replacing $M_{\Sigma_{c}^{*}}-M_{\Sigma_{c}}$ with $M_{\Sigma_{b}^{*}}-M_{\Sigma_{b}}$ where appropriate. Hence, the E2 component is reduced in the b-systems by a factor of $\sim M_{c} / M_{b}$, while the M1 component is essentially unchanged.

In conclusion, we have computed the leading contribution to the E2/M1 mixing ratio for the radiative decays $\Sigma_{c}^{*} \rightarrow \Lambda_{c} \gamma$. We find that the $1 / M_{c}$ suppression of the E2 amplitude is compensated for by a small energy denominator arising from the infrared behaviour of pion loop graphs in chiral perturbation theory. This indicates that the E2/M1 mixing ratio could be a few percent, depending on the $\Sigma_{c}^{*}-\Sigma_{c}$ spin symmetry breaking mass splitting. With fixed target experiments dedicated to charmed hadrons due to come on line within the next few years it is possible that this mixing ratio will be determined and compared with our estimates. The corresponding quantity in the b-baryon system will be reduced by a factor of $\sim M_{c} / M_{b}$.

I would like to thank J. Russ and M. Procario for useful discussions and bringing my attention to the possibility of measuring the E2/M1 mixing ratio. I would also like to thank P. Geiger for useful discussions. This work is supported in part by the Department of Energy under contract DE-FG02-91ER40682 and in part by the NSF under grant PHY8904035. 


\section{References}

[1] M.N. Butler, M.J. Savage and R.P. Springer, Phys. Lett. 304B (1993) 353; Phys. Lett. 314B (1993) $122(\mathrm{E}+\mathrm{A})$.

[2] H.Y. Cheng etal, Phys. Rev. 47 (1993) 1030.

[3] P. Cho and H. Georgi, Phys. Lett. 296B (1992) 408;Phys. Lett. 300B (1993) 410(E).

[4] N. Isgur and M.B. Wise, Phys. Lett. 232B (1989) 113; Phys. Lett. 237B (1990) 527.

[5] E. Eichten and B. Hill, Phys. Lett. 234B (1990) 511.

[6] H. Georgi, Phys. Lett. 240B (1990) 447.

[7] M.B. Wise, Phys. Rev. 45 (1992) 2188.

[8] G. Burdman and J. Donohue, Phys. Lett. 208B (1992) 287.

[9] T.M. Yan etal, Phys. Rev. 46 (1992) 145.

[10] P. Cho, Phys. Lett. 285B (1992) 145.

[11] M.B. Wise, Lectures given at the CCAST Symposium on Particle Physics at the Fermi Scale, May 1993.

[12] S. Capstick, Phys. Rev. 46 (1992) 1965.

[13] Z. Guralnik, M. Luke and A.V. Manohar, Nucl. Phys. B390 (1993) 474.

[14] E. Jenkins, Phys. Lett. 315B (1993) 431.

[15] H.J. Rose and D.M. Brink, Rev. of Mod. Phys. 39 (1967) 306. 


\section{Figure Captions}

Fig. 1. The graph giving the leading contribution to the E2 matrix element for $\Sigma_{Q}^{*} \rightarrow$ $\Lambda_{Q} \gamma$. There is a cancellation between the $\Sigma_{Q}^{*}$ and $\Sigma_{Q}$ intermediate states that becomes exact as they become degenerate.

Fig. 2. The E2/M1 mixing ratio $\delta$ for $\Sigma_{c}^{*} \rightarrow \Lambda_{c} \gamma$ as a function of $M_{\Sigma_{c}^{*}}-M_{\Sigma_{c}}$ for $g_{3}=$ $\sqrt{3 / 2} g_{A}, g_{2}=-3 / 2 g_{A}$ and $g_{A}=1.25$. An imaginary part (dashed line) arises when $M_{\Sigma_{c}^{*}}-M_{\Sigma_{c}}>m_{\pi}$. 


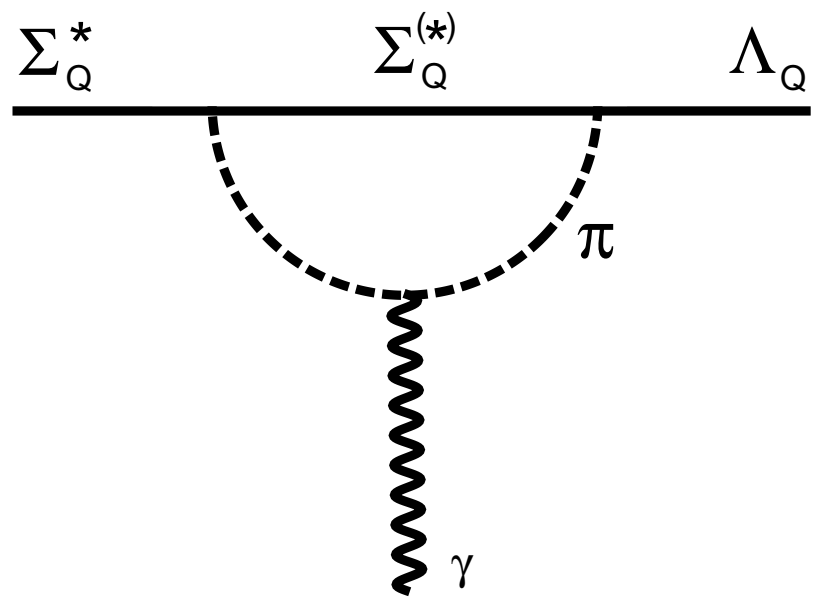

Figure 1

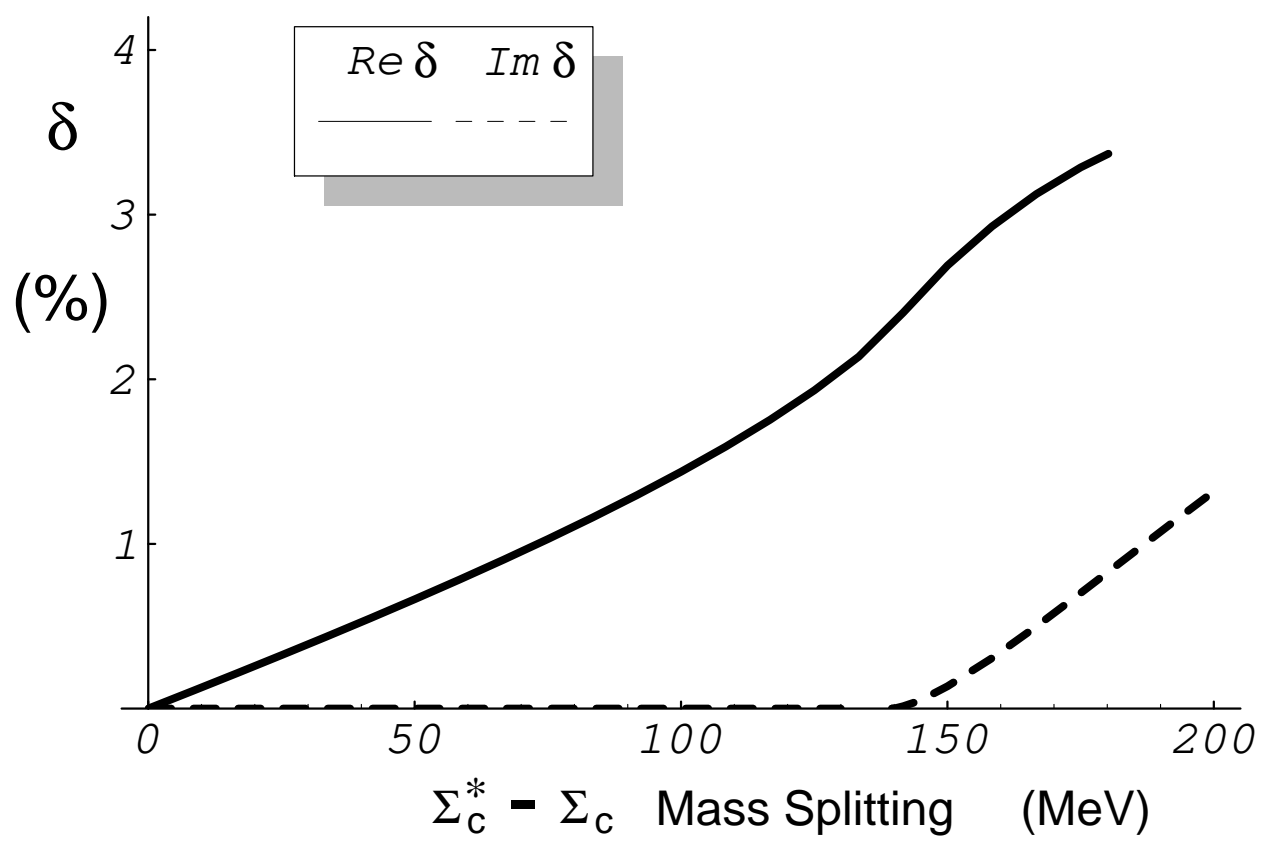

Figure 2 